\title{
Analgesic Effect of Intra-Articular Dexamethasone versus Fentanyl added as an adjuvant to Bupivacaine for Postoperative Pain Relief in Knee Arthroscopic Surgery Hazem El Sayed Moawad Weheba*, Mohamed Ahmed Sultan, Tawfik Abouhalawa Tawfik Hassan, Hosam I. El Said Saber \\ Department of Anesthesia and Surgical Intensive Care, Faculty of Medicine, Mansoura University, Egypt \\ *Corresponding author: Hazem El Sayed Moawad Weheba, Mobile: +201121516041 - +201012805817, E-mail: hazemmoawad@yahoo.com - hazemsayed@mans.edu.eg
}

\begin{abstract}
Background: Knee arthroscopy is usually associated with a variable degree of pain ranging from moderate-to-severe pain in about $70 \%$ of patients.

Objective: This trial was designed to assess the efficacy of intra-articular administration of dexamethasone versus fentanyl as adjuncts to bupivacaine in patients undergoing arthroscopic knee surgery.

Patients and methods: Eighty-nine patients of either sex were enrolled in this study. The patients were randomly divided into three equal groups. Group F that received intra-articular (IA) injection of $1 \mu \mathrm{g} / \mathrm{kg}$ fentanyl (In $2 \mathrm{ml}$ saline) added to $18 \mathrm{ml}$ of $0.25 \%$ bupivacaine, group D, which received IA injection of $8 \mathrm{mg}(2 \mathrm{ml})$ dexamethasone added to 18 $\mathrm{mL}$ of $0.25 \%$ bupivacaine and group $\mathrm{S}$ that received IA injection of $2 \mathrm{ml}$ normal saline added to $18 \mathrm{~mL}$ of $0.25 \%$ bupivacaine.

Results: The time required for the first request of analgesia in group F, group D, and group S was $5.7 \pm 0.7$ vs $4.5 \pm 0.5$ vs $3.3 \pm 0.5$ hours respectively. There were significant differences between both treatment groups and the control group $(\mathrm{p}<0.001)$ and in between both treatment groups $(\mathrm{p}<0.001)$ in favor of group $\mathrm{F}$. There was a significantly lower median visual analogue score in group $\mathrm{F}$ when compared to group $\mathrm{D}$ and $\mathrm{S}$ at 6 hours $(\mathrm{p}=0.006 \& 0.01$, respectively), 12 hours ( $p<0.001 \&<0.001$, respectively), and 18 hours $(p=0.003 \& 0.007$, respectively) postoperatively.

Conclusion: The addition of fentanyl or dexamethasone to IA bupivacaine in knee arthroscopic surgery provided a better quality of analgesia with less consumption of systemic analgesics without significant adverse effects.
\end{abstract}

Keywords: Knee arthroscopy, Fentanyl, Dexamethasone

\section{INTRODUCTION}

Knee arthroscopy is a minimally invasive surgical procedure commonly performed on a day-case basis. However, knee arthroscopy is usually associated with a variable degree of pain ranging from moderateto-severe pain in about $70 \%$ of patients following surgery ${ }^{(1)}$. Inadequately treated postoperative pain following knee arthroscopy results in delayed recovery, prolonged hospitalization, and increased medical care costs ${ }^{(2)}$. Several studies have been conducted in an attempt to find out an ideal analgesic technique that could be safe and satisfactory ${ }^{(1,3)}$. Intrathecal opioids, such as fentanyl or morphine, provide excellent postoperative analgesia but may cause side effects such as urinary retention, pruritus, nausea, and vomiting ${ }^{(4)}$. Anti-inflammatory drugs can provide good analgesia in the immediate postoperative period, and usually have more serious side effects ${ }^{(5)}$.

An intra-articular (IA) injection of local anesthetics and analgesics is a simple, effective, safe, and practical method in decreasing patients' postoperative pain in addition to avoiding the need for additional analgesics ${ }^{(6)}$. Bupivacaine is a local anesthetic that blocks peripheral afferent through blocking sodium ion channels. High lipid-solubility and large molecular size of bupivacaine result in slow absorption and longer duration of action ${ }^{(7)}$. However, to be an ideal analgesic, the drug must cover the completely postoperative period ( $\geq 24$ hours), therefore, bupivacaine is usually combined with many adjuncts to provide long-lasting postoperative analgesia ${ }^{(8)}$. The use of bupivacaine and opioids in conjunction was proved to act through different mechanisms. They have a synergistic action, thus providing longer analgesia. Fentanyl is a lipophilic opioid with a rapid onset, can prolong the duration of postoperative analgesia, and has less side effects in comparison with morphine when combined with bupivacaine ${ }^{(9)}$.

Dexamethasone is a potent, highly selective glucocorticoid that can inhibit the nociceptive transmission along the myelinated $\mathrm{C}$ fibers and when combined with local analgesics, it prolongs the duration of regional blocks ${ }^{(10)}$.

This randomized-controlled trial was designed to assess the efficacy and safety of intra-articular administration of dexamethasone versus fentanyl as adjuncts to the local anesthetic bupivacaine in patients undergoing arthroscopic knee surgery.

\section{PATIENTS AND METHODS}

This randomized double-blind study was conducted in the Knee Arthroscopic Surgery Unit, Mansoura University Hospital, from March 2019 to January 2020. Eighty-nine patients of either sex with ASA (American Society of Anesthesiologists) physical status I or II, aged from 20-65 years undergoing arthroscopic knee surgery were enrolled in this study.

\section{Ethical approval:}

The study was approved by the Institutional Review Board (code number MS.18.09.306), Faculty 
of Medicine, Mansoura University. The study was registered at clinical trials.gov with protocol ID NCT03847792. A written informed consent was taken from each patient. This work has been carried out in accordance with The Code of Ethics of the World Medical Association (Declaration of Helsinki) for studies involving humans.

Patients with contraindication to spinal anesthesia, patients with hemorrhagic or clotting dysfunction, allergy to the study drugs, patients on chronic pain therapy and patients refusing to participate in this study were excluded from the study.

\section{Preoperative management:}

All patients were assessed preoperatively by history taking, physical examination, electrocardiogram (ECG) and laboratory evaluation (complete blood count, liver and renal function tests, and coagulation profiles). The use of systemic analgesics pre- or intraoperatively were avoided. The day before surgery all patients were instructed how to use $10 \mathrm{~cm}$ visual analogue scale (VAS) for pain assessment. The scale consists of $10 \mathrm{~cm}$ horizontal line ranging from 0 (no pain) to 10 (worst imaginable pain) ${ }^{(11)}$. Patients were asked to mark the line vertically at a point, which matches their pain. Patients were informed about the details of the intraarticular analgesic injection and its possible benefits and complications. Written informed consent for the procedure was taken from all patients. All surgical procedures were performed under spinal anesthesia.

The patients were randomly divided into three equal groups (29 patients in each), using computer-generated randomization table. The patients and the administrator were blinded to the intra articular medications administered. The study solutions were prepared by an anesthesiologist who was blinded to the study.

The three groups according to intra-articular injected solution were: Group F: Patients received an IA injection of $1 \mu \mathrm{g} / \mathrm{kg}$ fentanyl (In $2 \mathrm{ml}$ normal saline) added to $18 \mathrm{ml}$ of $0.25 \%$ bupivacaine at the end of surgery. Group D: Patients received an IA injection of $8 \mathrm{mg}(2 \mathrm{ml})$ dexamethasone added to $18 \mathrm{~mL}$ of $0.25 \%$ bupivacaine at the end of surgery. Group S: Patients received an IA injection of $2 \mathrm{ml}$ normal saline added to $18 \mathrm{~mL}$ of $0.25 \%$ bupivacaine at the end of surgery.

\section{Intraoperative management:}

In the operating room, standard monitoring was applied to the patient including an electrocardiogram, pulse oximetry, and non-invasive blood pressure. Intravenous (IV) cannula 18-gauge was inserted, and an infusion of lactated Ringer's solution was started. Spinal anesthesia with $10 \mathrm{mg}(2 \mathrm{ml})$ hyperbaric bupivacaine $(0.5 \%)$ was performed at L3-4 intervertebral space through a midline approach with a 25 -gauge spinal needle with the patient in the sitting position. The patients were immediately placed in the supine position. The assessment of sensory block level was evaluated by the loss of the pinprick sensation using pin-prick test (needle prick). The assessment of motor block was done using a modified Bromage scale $(0=$ no motor block, 1 = inability to raise extended leg, able to bend knee, 2 = inability to bend knee, can flex ankle; and $3=$ no movement) ${ }^{(\mathbf{1 2})}$. Readiness to the surgery was defined as the presence of adequate motor block (Bromage's score $\geq 2$ ) and the loss of the pinprick sensation at L1 (lower back, hips, groin) on the operated side. Patients with incomplete or failed spinal anesthesia were subsequently excluded from the study and received general anesthesia.

\section{Technique of intra- articular injection:}

A pneumatic tourniquet was situated around the mid-thigh of the operative limb and was inflated to 100 $\mathrm{mmHg}$ above systolic pressure from the start of the procedure until 10 minutes after the analgesic solution injection. Near the end of the surgical procedure, the study solution (20 ml volume) was prepared and supplied in a coded syringe. The solution was injected once by the surgeon into the knee joint after suturing skin at the end of the surgical procedure through the drain then it was closed. After 10 minutes, the tourniquet was released and the drain was kept closed for about one hour.

\section{Postoperative care:}

Patients were monitored in the post-anesthesia care unit (PACU). The pain was reassessed and recorded by using visual analogue scale (VAS) at 1, 6, 12, 18, 24 hours postoperatively. Heart rate (HR) and mean arterial pressure (MAP) were recorded at the same times of pain assessment. When patients experienced pain (VAS score $\geq 4)$, a rescue dose of oral paracetamol $(1000 \mathrm{mg})$ was administrated and was repeated every 8 hours on patient demand. If the patients continued to have pain 30 minutes following paracetamol administration, meperidine $(20 \mathrm{mg})$ was given through intravenous route. The time to the first request for analgesia (the postoperative time/hr from intra-articular injection of the study solution until the patient started to ask for analgesia) was recorded. The total paracetamol and meperidine consumption during the first 24 hours after the operation was calculated. Any postoperative adverse events like nausea, vomiting, hypotension and shivering were also recorded during the 24 hours' study period.

\section{Outcomes of the study:}

The primary outcome of the study was the time to the first request for analgesia. Secondary outcomes included pain score using VAS, changes in hemodynamics (HR and MAP), the total dose of consumed analgesics, and the incidence of complications.

\section{Sample size calculation and statistical analysis:}

The sample size was calculated using Power Analysis and Sample Size software program (PASS) version 11.0.4 using a one-way ANOVA based on 
previously published results ${ }^{(3,13)}$ about the time to first request of analgesia as the primary outcome. Assuming that SD was 1 and the calculated effect size (f) was 0.43 , a sample size of 87 equally divided into three groups can achieve $95 \%$ power with a 0.05 significance level.

Statistical analysis of data was performed using SPSS $^{\mathrm{TM}}$ software, version 23 (Bristol, UK). Continuous variables were described as mean \pm standard deviation (SD) and discrete variables as median and range. Categorical variables were represented using numbers and proportions. One Way ANOVA and Repeated Measures ANOVA test were used to detect differences in the means of continuous variables. Mann-Whitney $U$ test was used to detect differences in the medians of discrete variables or non-parametric continuous variable, and Fisher exact test or Chi-square test were used for processing categorical variables. $\mathrm{P}$ values $\leq$ 0.05 were considered significant.

\section{RESULTS}

Regarding patients' selection, initially, one hundred patients who underwent arthroscopic knee surgery were assessed for eligibility. Five patients on chronic pain therapy, 4 patients with impaired coagulation, and 4 patients refusing the procedure, all were excluded from the study. A total of 87 patients were ultimately included to the study and the recruitment was halted once the desired patients were enrolled in the study (Figure 1).

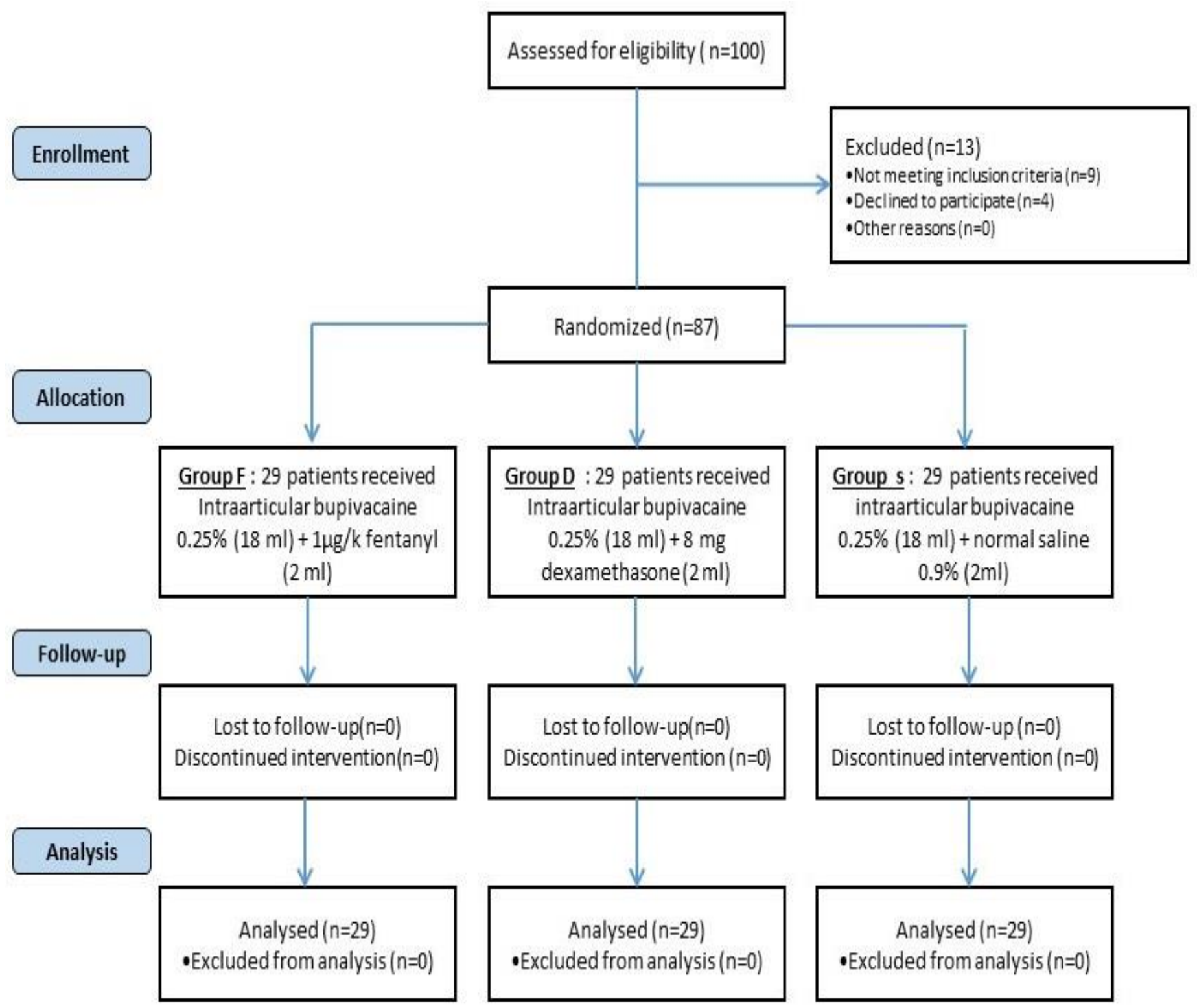

Figure (1): Flowchart demonstrating patients' selection and exclusion

About patients' characteristics, from the entire cohort $47 \%$ of patients were men. The mean age of patients was 30.5 \pm 7 years. The average BMI was $29.1 \pm 3.8 \mathrm{Kg} / \mathrm{m}^{2}$. Also, $50(57.5 \%)$ patients were ASA class I and $37(42.5 \%)$ were ASA class II. There were no significant differences between all groups concerning age, sex, BMI, and ASA class as shown in table (1). 
Table (1): Demographic data in the studied groups

\begin{tabular}{|c|c|c|c|c|c|}
\hline \multicolumn{2}{|c|}{ Variable } & Group F & Group D & Group $S$ & $P$ value \\
\hline \multicolumn{2}{|c|}{ Age (Years) } & $31.24 \pm 7.693$ & $28.76 \pm 6.457$ & $31.55 \pm 6.647$ & $\begin{array}{c}\mathrm{P}=0.5 \\
\mathrm{P} 1=0.53 \\
\mathrm{P} 2=1 \\
\mathrm{P} 3=0.39\end{array}$ \\
\hline \multirow[t]{2}{*}{ Sex } & Male & $62 \%(18)$ & $48 \%(14)$ & $52 \%(15)$ & $\mathrm{P}=0.64$ \\
\hline & Female & $38 \%(11)$ & $52 \%(15)$ & $48 \%(14)$ & $\begin{array}{l}\mathrm{P} 2=0.6 \\
\mathrm{P} 3=1\end{array}$ \\
\hline \multicolumn{2}{|c|}{ ASA (I: II) } & $15(52) / 14(48)$ & $18(65) / 11(35)$ & $29.68 \pm 4.219$ & $\begin{array}{c}\mathrm{p}=0.68 \\
\mathrm{p} 1=0.43 \\
\mathrm{p} 2=0.79 \\
\mathrm{p} 3=0.79 \\
\mathrm{P}=0.405 \\
\mathrm{P} 1=0.380 \\
\mathrm{P} 2=0.657 \\
\mathrm{P} 3=0.188\end{array}$ \\
\hline
\end{tabular}

Group F: Fentanyl Group, Group D: Dexamethasone Group, Group S: Saline Group. BMI: body mass index. $\mathrm{p}$ was considered significant when $<0.05$. P (between the three groups), P1 (group F VS group D), P2 (group F VS group S), P3 (group D VS group S).

Concerning primary outcomes, time to the first request of analgesia in group $\mathrm{F}$, group $\mathrm{D}$, and group $\mathrm{S}$ was $5.7 \pm$ 0.7 vs $4.5 \pm 0.5$ vs $3.3 \pm 0.5$ hours respectively as shown in table (2). There were significant differences between both treatment groups and the control group $(\mathrm{p}<0.001)$ and in between both treatment groups $(\mathrm{p}<0.001)$ in favor of group F.

Table (2): The time to first request for analgesia (hr.) and total Paracetamol (mg) and Meperidine (mg) consumption in the studied groups

\begin{tabular}{|c|c|c|c|c|}
\hline Variable & Group F (n=29) & Group D $(n=29)$ & Group S $(n=29)$ & P value \\
\hline $\begin{array}{l}\text { Time to first request for analgesia } \\
\text { (hours) }\end{array}$ & $5.69 \pm 0.660$ & $4.48 \pm 0.509$ & $3.34 \pm 0.484$ & $\begin{array}{l}\mathrm{p}<0.001^{*} \\
\mathrm{P} 1<0.001 * \\
\mathrm{P} 2<0.001 * \\
\mathrm{P} 3<0.001 *\end{array}$ \\
\hline Paracetamol (mg) & $827.59 \pm 804.85$ & $1448.28 \pm 869.57$ & $1655.17 \pm 897.45$ & $\begin{array}{l}\mathrm{p}<0.001 * \\
\mathrm{p} 1=0.022^{*} \\
\mathrm{p} 2=0.001 * \\
\mathrm{p} 3=1\end{array}$ \\
\hline Meperidine (mg) & $7.59 \pm 11.23$ & $7.59 \pm 13.54$ & $19.31 \pm 18.11$ & $\begin{array}{l}\mathrm{P}=0.003 * \\
\mathrm{P} 1=1 \\
\mathrm{P} 2=0.009 * \\
\mathrm{P} 3=0.009 *\end{array}$ \\
\hline
\end{tabular}

Group F: Fentanyl Group, Group D: Dexamethasone Group, Group S: Saline Group. p was considered significant when $<0.05$. P (between the three groups), P1 (group F VS group D), P2 (group F VS group S), P3 (group D VS group S).

Regarding secondary outcomes, postoperative pain VAS score showed no significant difference between studied groups at one hour \& 24 hours postoperatively as shown in table (3). On the other hand, there was a significantly lower median VAS in group F when compared to group D and $S$ at 6 hours $(p=0.006 \& p=0.01$ respectively), 12 hours $(p<$ $0.001 \& \mathrm{p}<0.001$ respectively), and 18 hours $(\mathrm{p}=0.003 \& \mathrm{p}=0.007$, respectively) postoperatively.

Table (3): Post-operative Visual analogue scale (VAS) score for pain assessment (0 -10) in the studied groups

\begin{tabular}{|l|l|l|l|l|}
\hline Time & Group F $(n=29)$ & Group D $(n=29)$ & Group S $(n=29)$ & P value \\
\hline
\end{tabular}




\begin{tabular}{|c|c|c|c|c|}
\hline One hour & $2(1-2)$ & $2(1-2)$ & $2(1-2)$ & $\begin{array}{l}\mathrm{P}=0.16 \\
\mathrm{P} 1=0.1 \\
\mathrm{P} 2=1 \\
\mathrm{P} 3=0.12\end{array}$ \\
\hline 6 hours & $2(1-3)$ & $2(2-3)$ & $2(2-5)$ & $\begin{array}{l}\mathrm{P}=0.02 * \\
\mathrm{P} 1=0.006^{*} \\
\mathrm{P} 2=0.01 * \\
\mathrm{P} 3=0.041^{*}\end{array}$ \\
\hline 12 hours & $2(1-2)$ & $3(1-3)$ & $2(2-3)$ & $\begin{array}{l}\mathrm{P}=0.001 * \\
\mathrm{P} 1=0.000^{*} \\
\mathrm{P} 2=0.000^{*} \\
\mathrm{P} 3=0.028^{*}\end{array}$ \\
\hline 18 hours & $1(1-2)$ & $2(1-2)$ & $2(1-2)$ & $\begin{array}{l}\mathrm{P}=0.01 * \\
\mathrm{P} 1=0.003 * \\
\mathrm{P} 2=0.007 * \\
\mathrm{P} 3=0.78\end{array}$ \\
\hline 24 hours & $2(1-4)$ & $2(1-5)$ & $2(1-3)$ & $\begin{array}{l}\mathrm{p}=0.17 \\
\mathrm{p} 1=0.65 \\
\mathrm{p} 2=0.07 \\
\mathrm{p} 3=0.17\end{array}$ \\
\hline
\end{tabular}

Group F: Fentanyl Group, Group D: Dexamethasone Group, Group S: Saline Group. p was considered significant when $<0.05$. P (between the three groups), P1 (group F VS group D), P2 (group F VS group S), P3 (group D VS group S).

Concerning postoperative hemodynamic changes, there were no statistically significant differences among the three groups regarding postoperative hemodynamic changes including both HR and MAP. Regarding HR, it showed a rise up to 6 hours postoperatively in the three study groups. After that, it started to decline in the three study groups as shown in figure (2). Regarding MAP, there were no significant changes during the follow-up period as shown in figure (3).

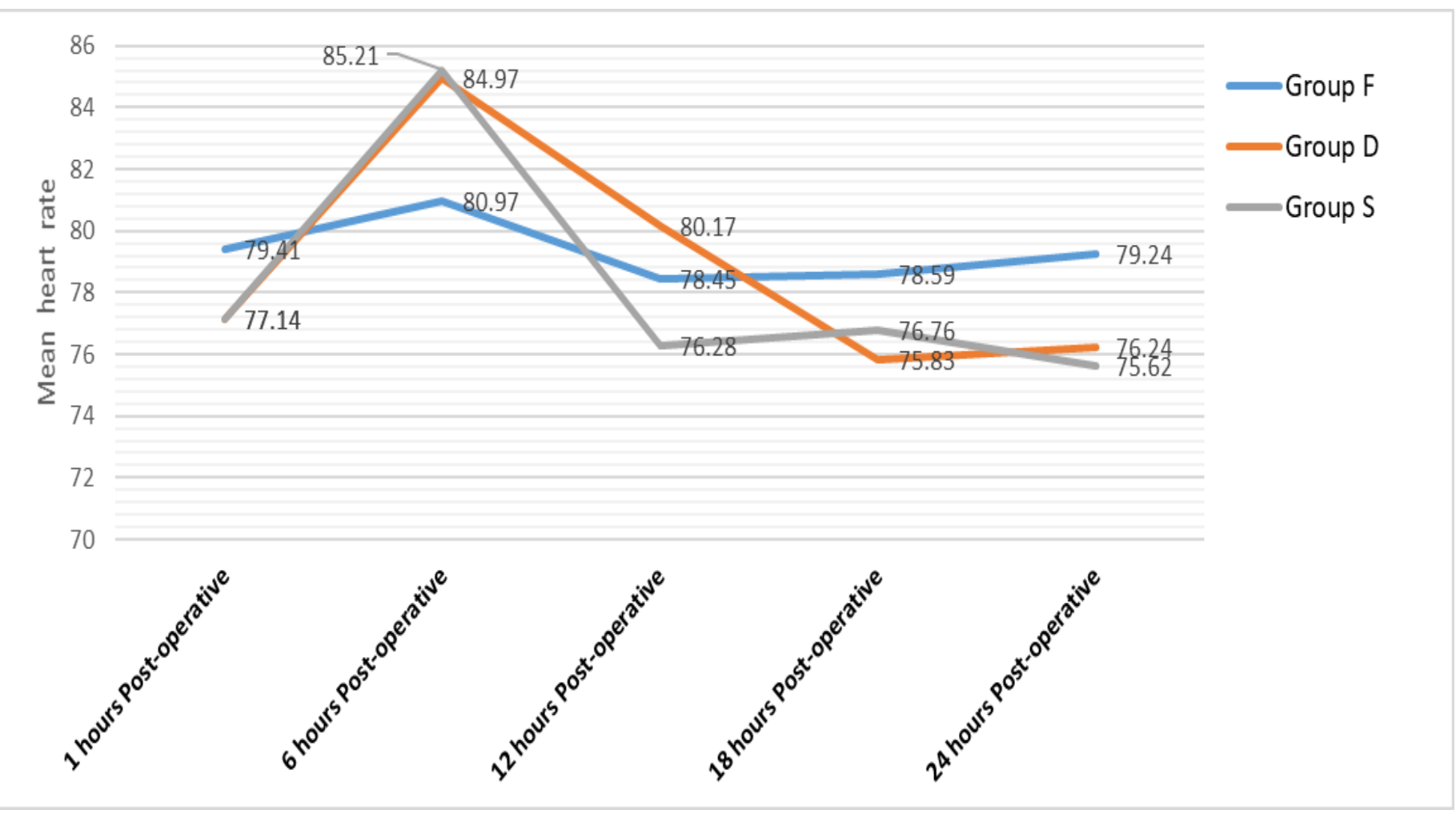

Figure (2): Postoperative heart rate (HR) changes over time intervals. 


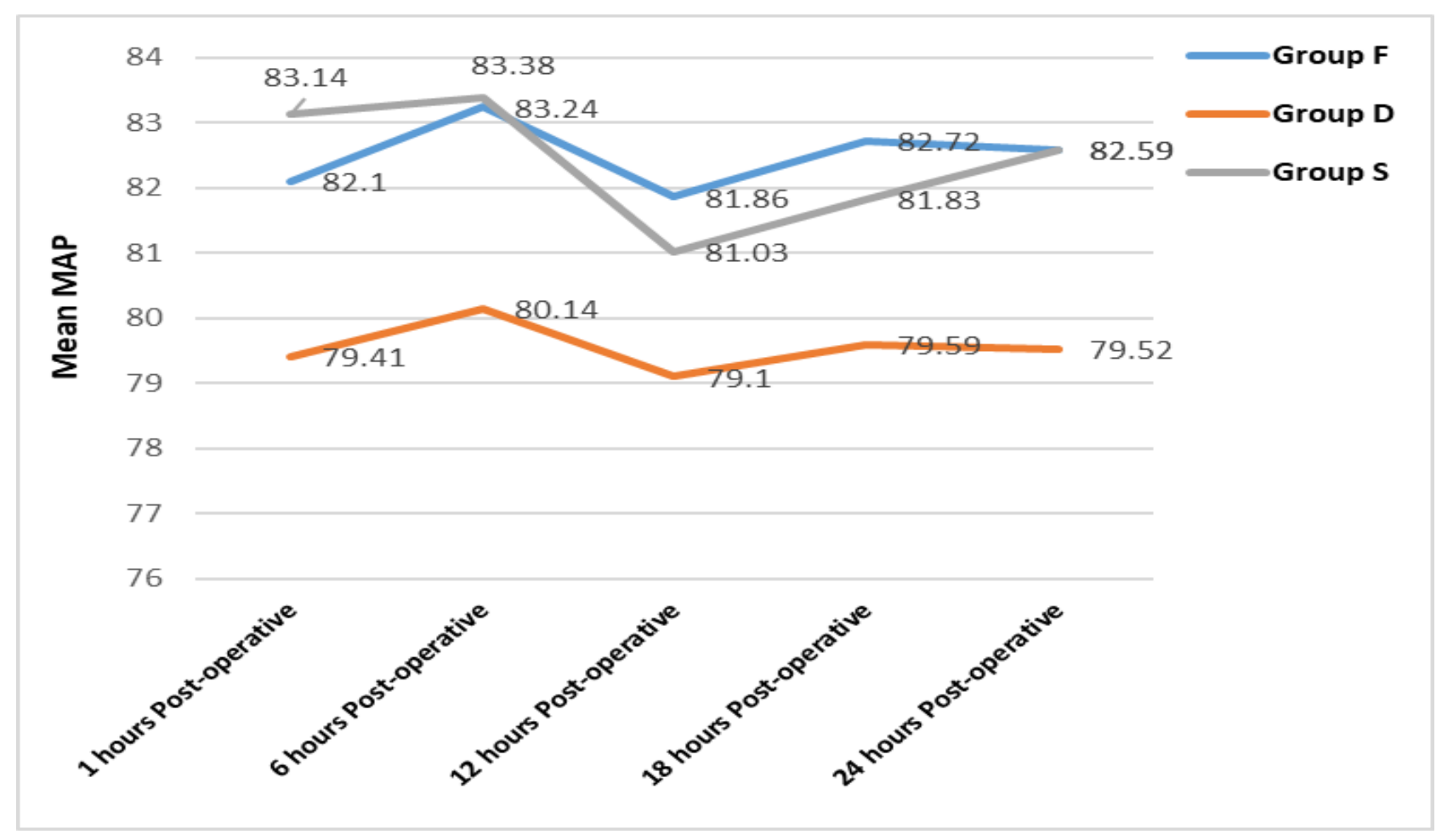

Figure (3) Postoperative mean arterial pressure (MAP) over time intervals.

About the total dose of postoperative $24 \mathrm{hr}$ consumption of paracetamol, it was significantly lower in group F (828 $\pm 805 \mathrm{mg})$ in comparison with group D (1448 $\pm 870 \mathrm{mg})$ and $\mathrm{S}(1655 \pm 897 \mathrm{mg})$ as shown in table (2). On the other hand, the total dose of postoperative $24 \mathrm{hr}$ consumption of meperidine was comparable between both treatment groups (group F and D) and significantly lower than group S as shown in table (2).

Regarding postoperative complications, eight patients $(9.1 \%)$ suffered from postoperative complications in the form of 3 patients (3.5\%) complained of nausea and vomiting, one patient (1.1\%) complained of hypotension, and 4 patients (4.6\%) complained from shivering as shown in table (4). It is important to notice that all these complications were minor in nature with no statistically significant difference between the study groups and required only conservative measures for proper control.

Table (4): Postoperative complications in the studied groups. Data were expressed as number and percentage.

\begin{tabular}{|l|c|c|c|l|}
\hline Variable & Group F (n= 29) & Group D (n= 29) & Group S (n= 29) & P value \\
\hline Nausea & $3 \%(1)$ & $0 \%(0)$ & $3 \%(1)$ & $\mathrm{P}=0.91$ \\
& & & & $\mathrm{P} 1=0.63$ \\
& & & & $\mathrm{P} 2=1$ \\
& & $0 \%(0)$ & $3 \%(1)$ & $\mathrm{P}=1$ \\
\hline Vomiting & $0 \%(0)$ & & $\mathrm{P}=1$ \\
& & & $\mathrm{p} 1=1$ \\
& & $0 \%(0)$ & $0 \%(0)$ & $\mathrm{p} 2=1$ \\
\hline Hypotension & $3 \%(1)$ & & & $\mathrm{P}=1$ \\
\hline Shivering & & & & $\mathrm{p} 1=1$ \\
& & $3 \%(1)$ & $\mathrm{p} 2=1$ \\
& & & $\mathrm{p} 3=1$ \\
\hline
\end{tabular}

Group F: Fentanyl Group, Group D: Dexamethasone Group, Group S: Saline Group. P was considered significant when $<0.05$. P (between the three groups), P1 (group F VS group D), P2 (group F VS group S), P3 (group D VS group S).

\section{DISCUSSION}


There is no consensus on a standard protocol to be used routinely in the control of post-arthroscopy knee pain, yet to be optimized. Needless to say that many previous studies reported improved analgesia after intra-articular injection of variable medications in arthroscopic knee surgeries ${ }^{(14)}$. Adjuncts or additives are often used with local anesthetics for their synergistic effect through prolonging the duration of sensory-motor block, limiting the cumulative dose requirement of local anesthetics, and extending the duration of analgesia ${ }^{(15)}$. Hence, this study was designed to evaluate the safety and efficacy of intra-articular dexamethasone versus fentanyl administered as an adjunct to the local anesthetic bupivacaine for postoperative analgesia in patients undergoing arthroscopic knee surgery. In the current study, fentanyl, dexamethasone, or normal saline were added to bupivacaine and were compared regarding the time to the first request for analgesia, HR, MAP, postoperative pain score, the total dose of postoperative systemic analgesics, and any adverse effects during the first postoperative $24 \mathrm{hr}$ of the study follow-up period.

Despite the previous investigation of the effect of the two treatment combination (bupivacaine + fentanyl or dexamethasone) ${ }^{(3,13)}$, it was important to design a three-armed design with its known sophistication to compare the results of the two treatment groups against a control group. Yet, still difficult to determine if the extra effect was due to added or synergistic effect of the adjuncts. The three study groups were adequately matched regarding all basic characteristics reflecting effective randomization. Also, the patients mostly belonged to the middle-aged group, situated in the area between overweight and obesity, and were classified as ASA class I or II. All the factors together ensured the safety of the protocol and optimized the results by avoiding the interference of other factors.

Although, it would be much accurate to use general anesthesia to assess postoperative pain, while in our study we adopted spinal anesthesia as it is the protocol followed in the knee arthroscopic unit. On the other hand, the use of systemic analgesics pre- or intraoperatively was avoided, so that the analgesic effect could be attributed to the study solution.

Previous studies either used injectable volume of 20,30 , or $40 \mathrm{ml}^{(3,13 \text {, and } 17)}$, higher volumes would be more difficult to be injected completely and may increase the intra-articular pressure resulting in increasing absorption and eliciting pain. In the current study, we used a $20 \mathrm{ml}$ volume, which ensured a higher concentration. Then, we compensated for the lost absorptive effect through the use of a tourniquet inflated for 10 minutes after injecting the study solution.

Regarding the primary outcome, the use of previously described combinations resulted in prolongation of analgesia time and delaying the request to analgesics. This time was 1.7 times longer in group $F$ vs 1.3 times in group $\mathrm{D}$, both when compared to the control group. The advantage of fentanyl over dexamethasone is that it is one of the most potent opioid analgesics a group of analgesics that was recently proved to produce potent receptor-specific analgesic effects outside the central nervous system. Opioid receptors are localized on peripheral terminals of sensory nerves and their endogenous ligands, opioid peptides, have been discovered in immune cells within inflamed tissue ${ }^{(18)}$. Besides, opioids (through kappa receptors) were proved to exert a potent antiinflammatory effect attenuating up to $80 \%$ of arthritis in a dose-dependent manner. This action had many mechanisms including a reduction of tumor necrosis factor and alteration in mRNA expression ${ }^{(19)}$.

The use of bupivacaine/fentanyl mixture resulted in a delay in analgesics request of about 5.7 hours, a result which is slightly higher than the results published by Mitra $\boldsymbol{e t}$ al. ${ }^{(20)}$ who reported a delay of about 5.2 hours despite the use of a $30 \mathrm{ml}$ of $0.25 \%$ bupivacaine and a fixed dose of fentanyl $(50 \mu \mathrm{g})$. This could be attributed to the use of general anesthesia instead of spinal anesthesia as in our study and the relatively lower fentanyl dose. On the contrary, bupivacaine/dexamethasone mixture result regarding the time to the first request of analgesia was about 4.5 hours, a result that is less than half the duration reported by Bhattacharjee and his colleagues ${ }^{(3)}$ (10.2 hours) despite the same mixture composition and the use of general anesthesia. This may be attributed to the repeated use of intravenous fentanyl on an hourly basis during the surgery. Similarly, this delay was far less than that reported by Moeen and coworkers ${ }^{(21)}$ who mentioned that patients did not require rescue analgesics during the whole follow-up period (3 days) despite the use of the same mixture composition and spinal anesthesia. This could be attributed to the routine use of t.i.d oral $1000 \mathrm{mg}$ paracetamol during the whole follow-up period.

In the three study groups, there was no noticeable effect on hemodynamics including both HR and MAP. These results are in concordance with those reported by Sayed and coworkers ${ }^{(22)}$ who fashioned a study on brachial plexus block using similar study solutions to ours in both treatment groups.

Regarding postoperative pain assessed using VAS, the fentanyl group was superior over other groups. The results of the fentanyl group are lower than those reported by Sayın and coworkers ${ }^{(23)}$ probably due to the added effect of spinal anesthesia in our study. On the other hand, our results tended to get higher starting from 6 hours compared to those reported by Mitra and coworkers ${ }^{(20)}$ despite larger bupivacaine in the latter study. In addition, the results of the dexamethasone group were higher than those reported by Moeen and coworkers (21) despite the similar protocol in both studies. On the contrary, these results are better than those reported by Bhattacharjee and coworkers ${ }^{(3)}$ who adopted general anesthesia in their protocol. 
Generally, the use of intra-articular bupivacaine with the adjuncts resulted in a decline of postoperative analgesic use compared to the control group. These results are in concordance with those reported in previous studies $(\mathbf{3}, \mathbf{1 3}, \mathbf{2 0}$, and $\mathbf{2 1})$.

This study was limited by its single-center nature, relatively small sample size, lack of measuring the pain score during walking, lack of assessment for any possible local tissue affection due to short followup, and failure to explain the nature of the added effect of the adjuncts. Future randomized trials are required to confirm the findings of this study.

\section{CONCLUSION}

The addition of fentanyl or dexamethasone as adjuncts to intra-articular bupivacaine injection in knee arthroscopic surgery provided a better quality of analgesia with less consumption of systemic analgesics. Moreover, more pain relief could be achieved using fentanyl rather than dexamethasone without any significant adverse effects.

Financial support and sponsorship: Nil. Conflict of interest: Nil.

\section{References:}

1. Xie D, Zeng C, Wang Y et al. (2015): A Single-Dose Intra-Articular Morphine plus Bupivacaine versus Morphine Alone following Knee Arthroscopy: A Systematic Review and Meta-Analysis. PLoS One, 10: 140512-16.

2. Rosseland L, Stubhaug A, Grevbo F et al. (2003): Effective pain relief from intra-articular saline with or without morphine $2 \mathrm{mg}$ in patients with moderate-to-severe pain after knee arthroscopy: a randomized, double-blind controlled clinical study. Acta Anaesthesiol Scand., 47: 732-738.

3. Bhattacharjee D, Biswas C, Haldar $P$ et al. (2014): Efficacy of intraarticular dexamethasone for postoperative analgesia after arthroscopic knee surgery. J Anaesthesiol Clin Pharmacol., 30 (3): 387-390.

4. Eroglu A, Saracoglu S, Erturk E et al. (2010): A comparison of intraarticular morphine and bupivacaine for pain control and outpatient status after an arthroscopic knee surgery under a low dose of spinal anaesthesia. Knee Surg Sports Traumatol Arthrosc., 18: 1487-95.

5. Rokhtabnak F, Ale Bouyeh M, Seyed Siamdust A et al. (2015): Comparison of the effects of intra-articular sole ropivacaine and combined ketorolac and ropivacaine for pain control after knee arthroscopy surgery. Br J Pain, 9: 149-156.

6. Sanel S, Arpaz O, Unay K et al. (2016): Comparison of intra-articular bupivacaine-morphine with bupivacainetenoxicam combinations on post-operative analgesia in patients with arthroscopic meniscectomy: a prospective, randomised study. Int Orthop., 40 (3): 601- 605.

7. Chahar P, Cummings K (2012): Liposomal bupivacaine: a review of a new bupivacaine formulation. J Pain Res., 5: 257-264.

8. Zeidan A, Kassem R, Nahleh $\mathrm{N}$ et al. (2008): Intraarticular tramadol-bupivacaine combination prolongs the duration of postoperative analgesia after outpatient arthroscopic knee surgery. Anesth Analg., 107: 292-299.

9. Korhonen A, Valanne J, Jokela $\mathrm{R}$ et al. (2003): Intrathecal hyperbaric bupivacaine $3 \mathrm{mg}+$ fentanyl $10 \mu \mathrm{g}$ for outpatient knee arthroscopy with tourniquet. Acta anaesthesiologica Scandinavica, 47: 342-346.

10. Liu J, Richman K, Grodofsky S et al. (2015): Is there a dose response of dexamethasone as adjuvant for supraclavicular brachial plexus nerve block? A prospective randomized double-blinded clinical study. Journal of Clinical Anesthesia, 27: 237-242.

11. Hawker G, Mian S, Kendzerska $T$ et al. (2011): Measures of adult pain: Visual analog scale for pain (vas pain), numeric rating scale for pain (nrs pain), mcgill pain questionnaire (mpq), short-form mcgill pain questionnaire (sf-mpq), chronic pain grade scale (cpgs), short form-36 bodily pain scale ( $\mathrm{sf}-36 \mathrm{bps}$ ), and measure of intermittent and constant osteoarthritis pain (icoap). Arthritis Care \& Research, 63:240-252.

12. Graham A, Mcclure J (2001): Quantitative assessment of motor block in labouring women receiving epidural analgesia. Anaesthesia, 56: 470-476.

13. Lu J, Fu J, Wang D et al. (2020): The efficacy of intraarticular fentanyl supplementation for knee arthroscopy: A meta-analysis of randomized controlled studies. Journal of Orthopaedic Surgery, 28: 1-7.

14. Terkawi A, Mavridis D, Sessler D et al. (2017): Pain Management Modalities after Total Knee ArthroplastyA Network Meta-analysis of 170 Randomized Controlled Trials. Anesthesiology: The Journal of the American Society of Anesthesiologists, 126: 923-937.

15. Swain A, Nag D, Sahu S et al. (2017): Adjuvants to local anesthetics: Current understanding and future trends. World Journal of Clinical Cases, 5: 307-11.

16. Jones K, Vojir C, Hutt E et al. (2007): Determining mild, moderate, and severe pain equivalency across painintensity tools in nursing home residents. J Rehabil Res Dev., 44: 305-314.

17. Yang Y, Zeng C, Wei J et al. (2017): Single-dose intraarticular bupivacaine plus morphine versus bupivacaine alone after arthroscopic knee surgery: a meta-analysis of randomized controlled trials. Knee Surg Sports Traumatol Arthrosc., 25 (3): 966-979.

18. Smith H (2008): Peripherally-acting opioids. Pain Physician, 11 (2): 121-132.

19. Walker J (2003): Anti-inflammatory effects of opioids. Adv Exp Med Biol., 521: 148-160.

20. Mitra S, Kaushal H, Gupta R (2011): Evaluation of analgesic efficacy of intra-articular bupivacaine, bupivacaine plus fentanyl, and bupivacaine plus tramadol after arthroscopic knee surgery. Arthroscopy, 27 (12): 1637-1643.

21. Moeen S, Ramadan I, Elkady H (2017): Dexamethasone and Dexmedetomidine as an Adjuvant to Intraarticular Bupivacaine for Postoperative Pain Relief in Knee Arthroscopic Surgery: A Randomized Trial. Pain Physician, 20 (7): 671-680.

22. Sayed M, Abd El-Hameed S, Ahmed E (2019): Comparative Study Between Dexamethasone and Fentanyl as An Adjuvant To Bupivacaine in Ultrasound Guided Supraclavicular Brachial Plexus Block in Upper Limb Surgeries. Al-Azhar Medical Journal, 48 (4): 501-512.

23. Sayın P, Dobrucalı H, Türk $H$ et al. (2015): Effects of intra-articular levobupivacaine, fentanyl-levobupivacaine and tramadol-levobupivacaine for postoperative pain in arthroscopic knee surgery. Acta Orthop Traumatol Turc., 49 (3): 267-273. 